great apprehension in performing and interpreting spirometry. The NICE (CG12) COPD guidelines state that all healthcare professionals managing patients with COPD should have access to spirometry and be competent in interpretation of results; and that it can be performed by any healthcare worker who has undergone appropriate training and keeps his/her skills up-to-date.

Aims We conducted an audit on junior doctors during their respiratory placement to establish whether hands-on skills training and teaching on spirometry in real-life may improve their understanding of the technique and reliability of interpreting spirometry.

Methods Doctors at different stages of training (foundation year (FY), core medical (CMT), GP vocational (GPVTS) and speciality trainees (ST)) participated in the audit. We used a pre-designed questionnaire containing a balanced mixture of questions testing procedural skill (maximum score 15) and interpretation (maximum score of 33) of different spirometry results. Confidence was assessed using a separate questionnaire of four domains. Baseline data was collected within the first month of joining the respiratory rotation, followed by training on technique and interpretation of results assisted by an accredited pulmonary physiologist (total time of 45 minutes). The same doctors were reassessed on the questionnaires in 12-16 weeks. Results 25 doctors completed the audit assessment (10 FY1, 5 FY2, 4 CT1, 2 CT2, 2 GPVTS and 2 ST3). Significant improvements from baseline were noted in the median (IOR) scores of performance of spirometry technique $(6(4,8)$ to $9(8,11) ; \mathrm{p}<0.001)$ and interpretation $(11.5(5,15)$ to $18(17,24.5) ; p<0.001)$. Moreover, there were marked improvements in total (performance and interpretation) and confidence scores from baseline.

Conclusion This audit demonstrates that spirometry is easily taught and its interpretation is a useful skill to acquire irrespective of a future career in respiratory medicine. We suggest that training for Foundation Year doctors is effective and feasible, and should be included in training programmes as spirometry is more reliable in the diagnosis and management of common respiratory conditions than PEFR testing mandated in the GMC core procedures.

\section{P240 ASSESSING THE ACCURACY AND CONFIDENCE OF CHEST X-RAY REPORTING BY MEDICAL DOCTORS}

doi:10.1136/thoraxjnl-2012-202678.301

IF Satia, S Bashagha, A Bibi, F Zaman, S Mellor. East Lancashire Hospitals NHS Trust, Blackburn, England

Introduction The reporting of CXRs for medical patients admitted to hospital vary across Europe. Our local policy is that all admission $\mathrm{x}$-rays are reported but this may not be available at the time of senior medical review on the assessment unit. Comparison between physicians and radiology reporting has suggested radiologists provide improved quality (1) and accuracy (2) of reporting. There are no current curriculum competencies for trainees nor is CXR reporting formally assessed in undergraduate or post-graduate examinations.

Aim To assess the accuracy and confidence of CXR reporting by all grades within the medical division for common chest diagnoses presenting to the medical assessment unit.

Method 10 CXRs were presented to all grades within the medical division with a short clinical history and one digital CXR. Doctors were asked to give a pre-test confidence (out of 5) in reporting CXRs and then also give individual confidence levels for each of their CXR diagnoses.

Results A total of 138 doctors completed the CXR quiz with average results and $95 \%$ confidence intervals shown in figure 1 . The least well answered CXR diagnoses were left lower lobe collapse (38\%), emphysema (45\%), and mediastinal widening (57\%). When correlated with average $\%$ confidence for each diagnosis, doctors were on average over-confident in the incorrect CXRs they answered; left lower lobe collapse (64\%), emphysema (62\%), bilateral pneumothoraces $(74 \%)$. Interestingly, for the correct diagnosis, the average confidence for diagnosing effusions, TB and pneumonia were much less. Conclusion SPRs and Consultants scored the highest marks with the highest average confidence levels. Junior trainees felt least confident about making their diagnosis and were less likely to be correct. We recommend that SPRs and consultants must review all the CXRs requested to ensure accuracy of diagnosis. There also needs to be discussion with the JRCPTB and educationalist about including CXR competency as part of a trainee's generic curriculum on the e-portfolio, something which is currently lacking.

\section{References}

1. Weiner SN. Radiology by non-radiologists: is report documentation adequate? Am J Manag Care. 2005 Dec; 11(12).

2. Zohair Al Aseri. Accuracy of chest radiograph interpretation by emergency physicians. Emergency Radiology, 2009; 16(2).

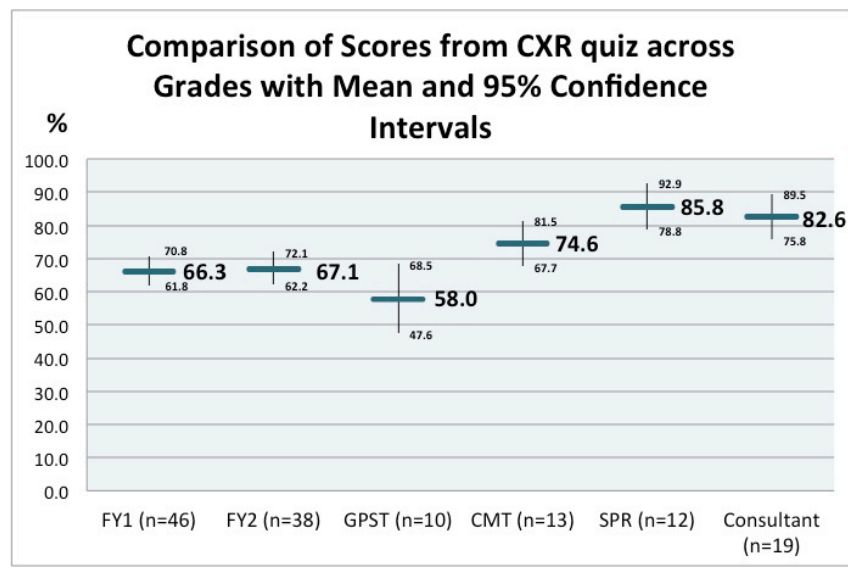

Abstract P240 Figure 1

\section{P241 PATIENTS' PERCEPTIONS OF THEIR RELATIONSHIP WITH HEALTHCARE PROFESSIONALS REGARDING THEIR ASTHMA MANAGEMENT: A UK SURVEY}

doi:10.1136/thoraxjnl-2012-202678.302

${ }^{1} \mathrm{M}$ Fletcher, ${ }^{2} \mathrm{D}$ Hiles, ${ }^{3} \mathrm{E}$ Luck. ${ }^{1}$ Education for Health, warwick, UK; ${ }^{2}$ Asthma UK, London, UK; ${ }^{3}$ NAPP Pharmaceuticals Limited, Cambridge, UK

Introduction and Objectives Asthma is one of the most common chronic conditions in the world, with 5.4 million individuals diagnosed in the UK alone. Here, we report data from a UK-based survey, conducted to assess how patients perceive the relationship with their general practitioner (GP) or nurse with regards to their asthma management.

Methods This was a cross-sectional online survey administered by YouGov plc (November 2011) to a panel of over 350,000 individuals. An e-mail was sent to panellists who had previously identified themselves as having asthma, inviting them to take part in the survey. Responses were collated and analysed by YouGov and Insight Research Group. The survey was completed by 1083 individuals; $49 \%$ were aged over 55 years and $45 \%$ were male.

Results Overall, 91\% of respondents reported that their asthma consultations occurred in GP practises; $69 \%$ had visited a practize nurse and $60 \%$ a GP. A total of $76 \%$ of respondents described the relationship with their healthcare professional (HCP) about their asthma management as 'good' or 'very good'. However, over onethird of respondents had either never been invited to (18\%), or had not always attended (17\%), asthma reviews. Moreover, $56 \%$ of respondents were unaware of Personal Asthma Action Plans and only $12 \%$ were currently using one, although $90 \%$ of those doing so found it 'very' or 'fairly' useful. The majority of individuals placed 